\title{
Mucocele of Appendix- A Detailed Case Report
}

\author{
Daspin D. ${ }^{1}$, Varsha Mary Khalkho ${ }^{2}$, Francis Gnanaprakasam³ ${ }^{3}$ Murali Krishnaswami ${ }^{4}$
}

${ }^{1}$ Radiologist, MIOT International Hospital, Chennai, Tamilnadu, India. ${ }^{2}$ Radiologist, MIOT International Hospital, Chennai, Tamilnadu, India. ${ }^{3}$ Senior Consultant, MIOT International Hospital, Chennai, Tamilnadu, India. ${ }^{4} \mathrm{Head}$ of the Department. MIOT International Hospital, Chennai, Tamilnadu, India.

\section{INTRODUCTION}

Mucocele of the appendix is rare; incidence is $0.2 \%-0.3 \%$ of appendectomy specimens and in $8 \%$ of appendiceal tumours. They typically occur in middle-aged patients. According to the literature there is discordant in its discussion of gender predominance.(1-3) The most common clinical manifestation is a palpable mass in the right lower quadrant of the abdomen. The patient can present either with or without pain. Some patients are asymptomatic, for such patients mucocele is found incidentally on imaging or during laparotomy or laparoscopy during other surgery. If a super infection is present, mucinous cystadenoma can be clinically indistinguishable from non-tumoral acute appendicitis.(4) In patients with a mucocele, the risk of developing an adenocarcinoma of the colon is six times greater than general population.(2) We are presenting 4 cases with different spectrum of manifestations in our institute. All the patients presented in our institute presented with abdominal pain followed by CT scan with appendicectomy. The histopathological correlation was also made from the sample and was confirmed in the follow up.

\section{PRESENTATION OF CASE}

\section{Case 1}

A 65-year-old gentlemen presented with history of vague abdominal pain in the right iliac fossa for 6 months. No other aggravating features were noted. Initial ultrasound showed a long tubular appendix from the base of caecum. Few calcific areas were also noted within it.

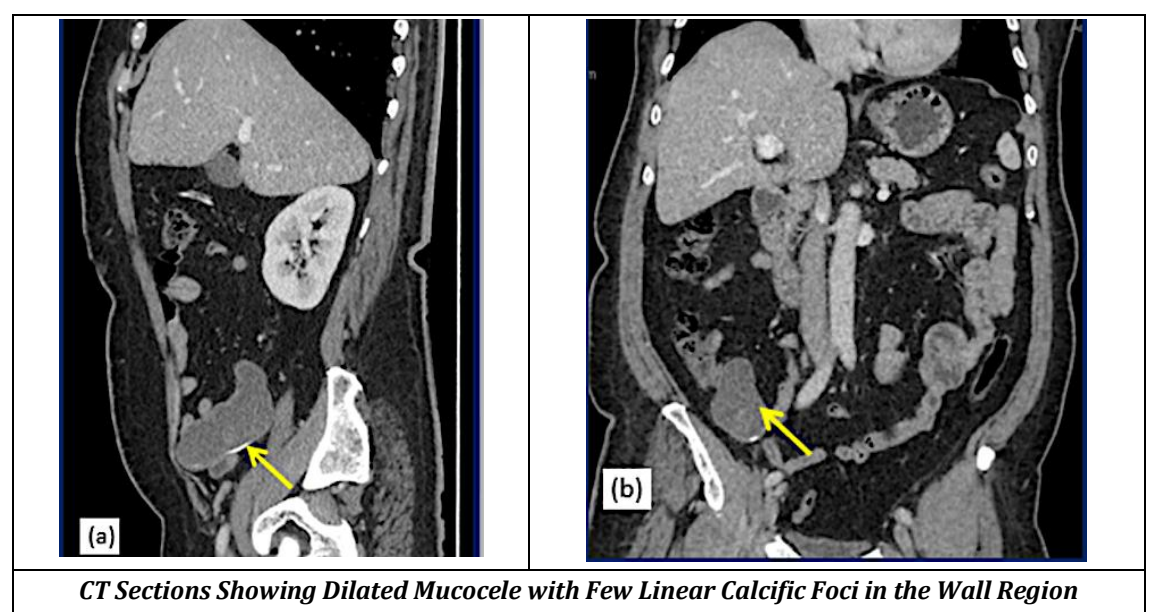

Corresponding Author: Dr. Daspin $D$.,

Thawa Building and Tower, 13J/2, Virugambakam, Chennai, Tamilnadu, India. E-mail: daspins@yahoo.com

DOI: $10.14260 /$ jemds/2019/690

Financial or Other Competing Interests: None.

How to Cite This Article:

Daspin D, Khalkho VM, Gnanaprakasam F, et al. Mucocele of appendix- a detailed case report. J. Evolution Med. Dent. Sci. 2019;8(42):3182-3184, DOI: $10.14260 / \mathrm{jemds} / 2019 / 690$

Submission 14-12-2017, Peer Review 21-09-2019, Acceptance 27-09-2019, Published 21-10-2019.

\section{(c) (i) $($ ) $\Theta$}


CT scan of the patient confirmed the findings. A tubular fluid filled appendix from base of caecum with mural calcifications noted. Diagnosis of mucocele of appendix was made. Patient was taken for surgery under laparoscopic guidance. Large mucocele of the appendix with thickened base of the appendix was noted. Side - side ileocolic anastomosis was done using two staples and the appendix was resected. Histopathology of the appendix and terminal ileum showed no significant abnormality.

\section{Case 2}

A 61-year-old female patient with complaint of progressive breathlessness, heaviness of chest, fever on and off vague pain in the abdomen for 1 month. Patient was analysed for tuberculosis. Left thoracocentesis was done and strawcoloured fluid was aspirated. Based on the cytology, pleural fluid was exudate with mononuclear cells and high ADA. No malignant cells were detected. Based on this the patient was on ATT. Incidentally USG Abdomen showed fluid filled lump the right lumbar region.

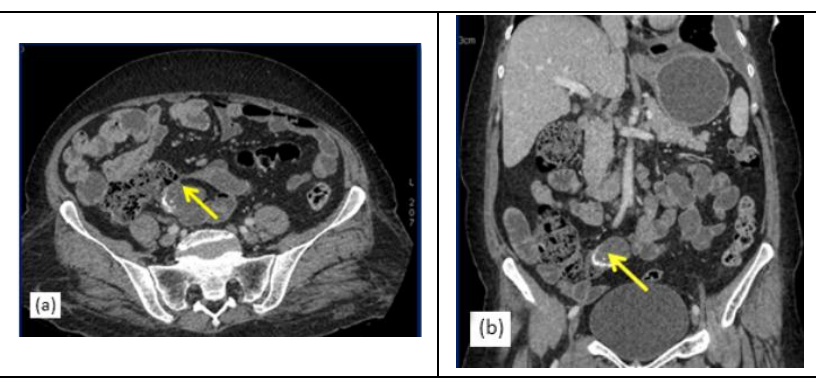

CT Sections Showing Mucocele with Thickened Walls and Few Curvilinear Calcific Foci in the Wall Region

CT Abdomen- A 4.0 × $2.9 \mathrm{~cm}$ cystic lesion with thickened wall and calcifications was seen in the RIF adjacent to caecum. The appendix was not separately seen. Based on these findings, possibility of mucocele of appendix was made. Patient was managed conservatively with ATT and is in follow up for mucocele of appendix.

\section{Case 3}

A 73-year-old male presented with a complaining of abdominal pain predominantly involving the right iliac fossa region. Patient also presented with vomiting and cramps associated within it. Initial USG Abdomen showed a large cystic lesion in the right iliac fossa region. Associated liver showing fatty changes and caudate lobe hypertrophy.

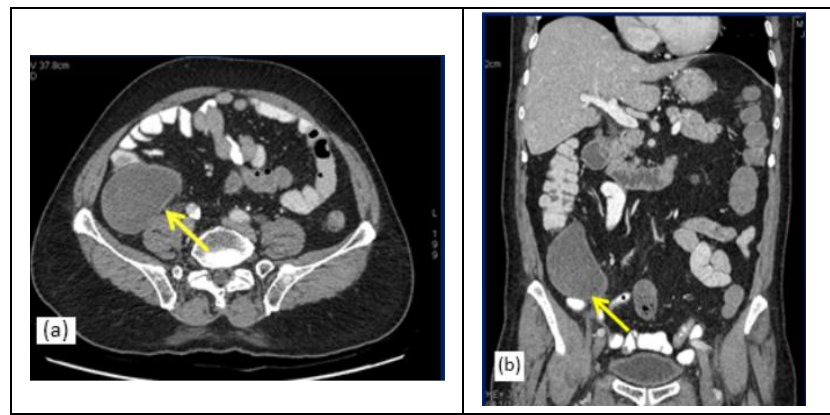

CT Sections Showing Chronic Liver Disease with Large Thin Walled Cystic Lesion (Mucocele) in the Right Iliac Fossa Region
CT Abdomen- Large cystic lesion with thin wall was seen in right iliac fossa region measuring $8 \times 5.4 \mathrm{~cm}$. Liver shows caudate lobe hypertrophy with ill-defined non enhancing hypo-dense lesions are noted in the segment VII of liver. Based on these findings, possibility of mucocele of appendix was made. Patient was taken for surgery followed by right transverse incision was made. There was a mass present in the right ileocaecal junction. Colon was mobilised from ileocecal junction to transverse colon and excision of the mass was done. Histopathology was diagnosed as low-grade mucinous neoplasm of appendix.

\section{Case 4}

A 50-year-old male was admitted with complaint of fever and abdomen pain for $21 / 2$ months for evaluation. History of anorexia and weight loss for 3 months. CT scan done showed features of gross ascites with scalloping of liver with omental, calcific, peritoneal and lymph nodal deposits. Large cystic lesion was noted in the RIF. The appendix was not separately visualized from the lesion. Based on the findings pseudomyxoma peritonei was diagnosed. Patient was taken for exploratory laparotomy and omentectomy with subtotal gastrectomy done. Subtotal colostomy and peritoneal resection with Roux-en-Y gastrojejunostomy and ileostomy anastomosis was done.

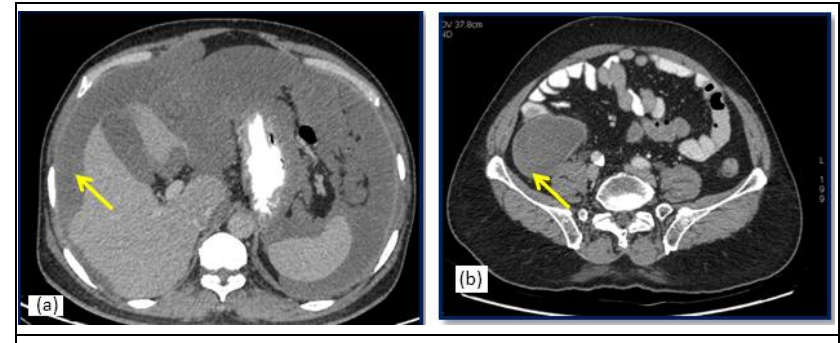

CT Sections Showing Features of Pseudomyxoma Peritonei with Dilated Mucocele and Gross Ascites Causing Scalloping of Liver Parenchyma

Findings- Extensive disease involving whole of omentum with large mucinous mass around the appendix was made. Disease involving the caecum, colon, diaphragmatic peritoneum, liver, spleen and greater curvature of stomach. Histopathological examination from the peritoneum showed features of pseudomyxoma peritonei.

\section{DISCUSSION}

The term mucocele is simply a macroscopic description of an appendix that is grossly distended by mucus. They can result from chronic appendiceal obstruction that may be caused either by benign or malignant lesions. These are thin walled, usually about 3-6 cm in diameter. Sometimes the mucocele can manifest as an enormous tumour up to $25 \mathrm{~cm}$ in size; in such cases, it is called a giant mucocele. $(5,6)$

Mucocele can manifest with four histologic subtypes: a simple mucocele, focal or diffuse mucosal hyperplasia, mucinous cystadenoma, and mucinous cystadenocarcinoma. A simple mucocele or retention cyst exhibits degenerative changes of the epithelium with no epithelial atypia. The degenerative changes could be due to obstruction, which can be caused by an appendicolith, postoperative scarring following appendicitis, appendiceal carcinoma, carcinoma of 
cecum, or appendiceal volvulus. This is found in approximately $20 \%$ of cases.(3) Focal or diffuse hyperplasia causes mild dilatation, this histologic subtype is seen in $20 \%$ of cases.(3,5) Mucinous cystadenoma, which represents a benign neoplasm, is the most common type of mucocele, accounting for about $50 \%$ of cases.(5) In about $20 \%$ of cases, they may perforate with mucus seeding, which leads to pseudomyxoma peritonei. Malignant mucocele has increased risk for perforation with peritoneal implantations, which also causes pseudomyxoma peritonei.(7) A rare variant of the mucocele is myxoglobulosis with multiple small intraluminal globules. These globules may calcify and produce $1-10-\mathrm{mm}$ mobile calcifications. ${ }^{(8)}$

On US images, the mucocele appears anechoic or hypoechogenic in right iliac fossa. In some cases, acoustic shadowing from calcifications is seen. Further characterization of lesion by CT or MRI is indicated. The anatomic relationship between the tumour and cecum is usually more obvious on CT and MR images than it is on US images. CT is more sensitive than MR imaging for detecting mural calcifications. Also, to rule out other pathology which can mimic mucocele like cystic ovarian neoplasm or tuboovarian abscess, peri-appendiceal abscess, enteric duplication cyst, mesenteric cyst, or hydrosalpinx CT and MRI is superior to USG. At CT, the lesions appear homogeneous and cystic. They are well circumscribed with central areas of low attenuation value.(2) The diameter is more than $13 \mathrm{~mm}$. In about $50 \%$ of cases, there are calcifications of the appendiceal wall; this feature is suggestive of a mucocele.

On magnetic resonance (MR) images, the tumour is hyperintense on T2-weighted pulse sequences. Depending on the mucin concentration, the signal may be variably hypointense to isointense on T1-weighted images. Soft tissue thickening, infiltration of the perifocal fat, and an irregular mucocele wall are signs of malignancy, secondary inflammation, or both, if seen on CT scan or MRI.

A mucocele may act as the lead point for ileocolic intussusception. Other complications such as torsion with gangrene and haemorrhage can arise. If a mucocele ruptures, it can cause a diffuse intraperitoneal accumulation of gelatinous material called pseudomyxoma peritonei, which is potentially lethal. The treatment of choice for a mucocele is appendectomy. During surgery, it is essential to inspect the whole abdomen because mucoceles are associated with an increased risk of colonic neoplasms and ovarian tumours.(2) Because apparently benign lesions can undergo a malignant transformation, a nonsurgical treatment is unacceptable. For mucinous cystadenocarcinoma, right hemicolectomy is usually needed, whereas for hyperplasia and cystadenoma, appendicectomy usually suffices if the resection margins are free. For mucinous tumours of uncertain malignant potential and low-grade mucinous tumour as well as pseudomyxoma peritonei, the decision is not as simple.
The mean 5-year survival rate in patients with pseudomyxoma peritonei is $25 \%-65 \%$, whereas patients without mucus seeding have an excellent prognosis. Most of the deaths caused by pseudomyxoma peritonei is due to intestinal obstruction. $(9,10)$ The treatment for pseudomyxoma peritonei is cytoreductive surgery with a debulking procedure and intraperitoneal chemotherapy.

\section{CONCLUSIONS}

Mucocele of appendix should be considered in the differential diagnosis of cystic lesions in the right lower abdomen. Imaging helps in diagnosis and determining the surgical approach. Mucocele of appendix should be surgically resected to avoid any malignant transformation of benign lesion or rupture leading to pseudomyxoma peritonei, as in many cases underlying small malignancy cannot be ruled out on imaging. The final diagnosis of a lesion being benign or malignant, is through histopathology.

\section{REFERENCES}

[1] Macek D, Jafri SZ, Madrazo BL. Ultrasound case of the day: mucocele of the appendix. RadioGraphics 1992;12(6):1247-9.

[2] Federle MP, Anne VS. Mucocele of the appendix. In: Federle MP, edr. Diagnostic imaging: abdomen. Salt Lake City, Utah: Amirsys 2004: p. 26-7.

[3] Ruiz-Tovar J, Teruel DG, Castiñeiras VM, et al. Mucocele of the appendix. World J Surg 2007;31(3):542-8.

[4] Roberge RJ, Park AJ. Mucocele of the appendix: an important clinical rarity. J Emerg Med 2006;30(3):303-6.

[5] Persaud T, Swan N, Torreggiani WC. Giant mucinous cystadenoma of the appendix. RadioGraphics 2007;27(2):553-7.

[6] Francica G, Lapiccirella G, Giardiello C, et al. Giant mucocele of the appendix: clinical and imaging findings in 3 cases. J Ultrasound Med 2006;25(5):643-8.

[7] Lim HK, Lee WJ, Kim SH, et al. Primary mucinous cystadenocarcinoma of the appendix: CT findings. AJR Am J Roentgenol 1999;173(4):1071-4.

[8] Gonzalez JE, Hann SE, Trujillo YP. Myxoglobulosis of the appendix. Am J Surg Pathol 1988;12(12):962-6.

[9] Isaacs KL, Warshauer DM. Mucocele of the appendix: computed tomographic, endoscopic and pathologic correlation. Am J Gastroenterol 1992;87(6):787-9.

[10] Pickhardt PJ, Levy AD, Rohrmann CA Jr, et al. Primary neoplasms of the appendix: radiologic spectrum of disease with pathologic correlation. RadioGraphics 2003;23(3):645-62. 